\title{
BMJ Open Understanding and addressing the challenges of conducting quantitative evaluation at a local level: a worked example of the available approaches
}

\author{
Sebastian Hinde (D) , Laura Bojke, Gerry Richardson
}

To cite: Hinde S, Bojke L, Richardson G. Understanding and addressing the challenges of conducting quantitative evaluation at a local level: a worked example of the available approaches. BMJ Open 2019;9:e029830. doi:10.1136/ bmjopen-2019-029830

- Prepublication history and additional material for this paper are available online. To view these files, please visit the journal online (http://dx.doi org/10.1136/bmjopen-2019029830).

Received 13 February 2019 Revised 30 October 2019 Accepted 31 October 2019

\section{Check for updates}

(C) Author(s) (or their employer(s)) 2019. Re-use permitted under CC BY-NC. No commercial re-use. See rights and permissions. Published by BMJ.

Centre for Health Economics, University of York, York, UK

Correspondence to Mr Sebastian Hinde; sebastian.hinde@york.ac.uk

\section{ABSTRACT}

Objectives In the context of tightening fiscal budgets and increased commissioning responsibility, local decisionmakers across the UK healthcare sector have found themselves in charge of the implementation and evaluation of a greater range of healthcare interventions and services. However, there is often little experience, guidance or funding available at a local level to ensure robust evaluations are conducted. In this paper, we evaluate the possible scenarios that could occur when seeking to conduct a quantitative evaluation of a new intervention, specifically with regards to the availability of evidence. Design We outline the full set of possible data scenarios that could occur if the decision-maker seeks to explore the impact of the launch of a new intervention on some relevant quantifiable outcomes. In each case we consider the implicit assumptions associated with conducting an evaluation, exploring possible situations where such scenarios may occur. We go on to apply the scenarios to a simulated dataset to explore how each scenario can result in different conclusions as to the effectiveness of the new intervention.

Results We demonstrate that, across the full set of scenarios, differences in the scale of the estimated effectiveness of a new intervention and even the direction of effect are possible given different data availability and analytical approaches.

Conclusions When conducting quantitative evaluations of new interventions, the availability of data on the outcome of interest and the analytical approach can have profound effects on the conclusions of the evaluation. Although it will not always be possible to obtain a complete set of data and conduct extensive analysis, it is vital to understand the implications of the data used and consider the implicit assumptions made through its use.

\section{INTRODUCTION}

Clinical commissioning groups (CCGs), local authorities, and other local decision-makers are under increasing pressure to demonstrate the value of any newly commissioned activities given tightening fiscal budgets. Although the Health and Social Care Act of 2012 was instrumental in allowing local decisionmakers to be responsive to the health needs of the population they serve, it provided
Strengths and limitations of this study

- Highlights the risks of partial analysis of time series data used to evaluate the impact of a service.

- Presents the assumptions implicitly made through the differential use of data to inform quantitative evaluation in a range of scenarios.

- Demonstrates that even a well-designed analysis is constrained by the available data.

- Provides guidance aimed at local decision-makers, who are typically overlooked in the published methodological guidance.

- The use of simulated data allows for a clear demonstration of the scenarios but risks oversimplifying the nature of 'real-world' data.

little guidance on how to do so in an effective and cost-effective manner. As a result, local decision-makers have found themselves caught between two worlds, neither being served by national evidence generation due to the decentralisation of funding, nor with the ability, finance or structure to generate robust evidence, such as randomised trials.

Although collaborations between the Local Government Association, Department of Health, National Health Service (NHS) England and others have led to a number of guides for good evaluation and evidence generation, ${ }^{2-4}$ these have had a broad focus on the theory of good research, rather than offering practical advice for analyses.

Although in some cases, such as the Vanguard projects, ${ }^{4}$ funding has been ringfenced for evaluation, it is more common that the decision to conduct a service evaluation by local decision-makers comes at the detriment of the service provision itself. As a consequence, any evaluation may be limited in scope, and the ability to fund sufficiently robust data collection severely compromised. Although there are inevitably risks of funding services based on inadequate evidence, as we 
will go on to demonstrate, there is little logic in funding sophisticated studies that threaten the provision of the service itself.

It has been the experience of these authors (GR is the University of York representative on York Teaching Hospital's Council of Governors; GR and LB are members of the Vale of York CCG's Research Group; and GR, LB and $\mathrm{SH}$ have experience in evaluating a number of local interventions including the Harrogate and District CCG's Vanguard programme, a Core 24-hour mental health liaison service, and Tier $3 \mathrm{wt}$ loss services) that these factors have resulted in either no quantitative evaluation of new service provision or evaluations that are based on limited interpretations of outcome measures and incomplete data collection. This is despite the move towards monitoring of services, both for quality and financial reasons, and falls in the cost of data generation, which have meant its collection and use is no longer an insurmountable barrier to evaluation.

In this paper, we explore a range of different scenarios faced by a local decision-maker depending on the availability of data and the analytical approach taken. We go on to use a stylised case study to explore the implications of each scenario on the estimated impact of the intervention and the likely conclusions. We focus on a quantitative evaluation but highlight the importance of a mixedmethod approach in achieving a robust evaluation.

We take as a starting point a decision-maker who is seeking to evaluate a new intervention, where intervention is used to describe any new or change in service, care pathway or treatment. They possess time series data on an outcome of interest over a series of time points, which is hypothesised to be impacted by the new intervention. These data may be at an aggregated level (eg, local population) or data for individuals (eg, patients or households). Such a generalised situation is common, with the decision-maker being anything from CCGs, c authorities, to mental health providers. Although the possible set of outcomes of interest is wide, the need to generalise findings often results in focus being on broad process outcomes, such as non-elective attendances, and length of stay, which are easily benchmarked. Such an analysis is expected to play a role in a decision-making process informed by a number of other quantitative and qualitative considerations.

\section{DIFFERENT SCENARIOS}

In this section, we consider the full set of data scenarios and analytical approaches that may occur when seeking to evaluate the impact of the launch of a new intervention on a single outcome of interest. We explore the range of implicit assumptions that are made for each of the scenarios, and possible examples of how each may occur. The different cases are characterised as six overarching scenarios. It is the experience of these authors that it is most common for evaluation of an intervention to be done retrospectively or towards the end of a project, primarily due to a lack of evaluative experience and funding to embed evaluation from an early stage; however, there is a lack of reviews of the methodology applied by local decision-makers in such setting.

\section{Scenario 1: follow-up data but no prelaunch data for the intervention area}

In its simplest form an evaluation may consist of only data collected after the launch of intervention with no historical evidence, for example if the intervention was unplanned and data could not be collected retrospectively, such as a piece of hospital infrastructure being replaced. Such an analysis can therefore only comment on the trajectory of the data over the intervention period as there is no knowledge of the counterfactual (what would have happened had the intervention not occurred), and no data on which to base any estimation. If any estimation of the total impact of the intervention is required, assumptions or external evidence would be required to inform the counterfactual.

\section{Scenario 2: follow-up data and a single prelaunch data point for the intervention area}

Second, we consider a situation where the decision-maker has only historical data for the final period before the launch of an intervention. Such a situation may occur when the decision to conduct an evaluation occurs only a short time before the launch and data cannot be collected retrospectively. Depending on the aggregation and availability of data two subscenarios are available:

A. Data are only available for the last period before launch and a single time point of the postlaunch time series, a simple before and after the statement is possible. In all cases, some implicit or explicit statement is beneficial regarding the generalisability of the observed data and trends in the data over the intervening time period. Such a case would occur if data were only available at set time points and only informative of a short time period, for example, annually occurring surveys or audits.

B. Data are available for the last period before launch and all post-intervention time points, allowing an average change over the period from the first time point to be calculated with some additional knowledge of how the data changed over the period. This might occur if repeated data collection is possible prospectively, such as the collection of electronic patient data once relevant patients have been identified and consented.

Given the limited prelaunch data available in this scenario, we must assume that, had the intervention not been launched, the outcome would have stayed at the same level as in the last time point before launch. Although this assumption is inevitable if no other data are available, it risks being misleading if there is some underlying trend in the outcome, or if it is subject to natural variation from one time point to the next. 
Scenario 3: data are available covering the full prelaunch and postlaunch period for the intervention area

To overcome the limitations of scenario 2, historical data in the intervention area can be used to inform the baseline value and any underlying trends in the outcome over time by relaxing the assumption that outcome data would have remained static. As with scenario 2, alternative aggregation of the historical data can result in different implications:

A. Both prelaunch and postlaunch may only be available as average values aggregated over a long period, for example, if the data access is limited to annual audit figures that cover the entire prelaunch period. This scenario implies that no consideration of the disaggregated trends is possible.

B. Extensive disaggregated data are available both before and after the launch. This allows for the direct comparison of each postlaunch time period with some matched period in the preintervention data, for example, comparing January in one year with January in the next. The matching is used to conduct the analysis at a more disaggregated level, as well as adjusting for other factors such as seasonality and budgetary cycles. Although the average estimate of the impact of the intervention launch will be the same as part A, we now have the ability to investigate the change in trend over the time period. Such a case would occur either when an evaluation and data collection was started some time before the intervention launch, or when data on the outcome are readily available retrospectively. For example, if the evaluation is concerned with emergency department attendances over time, historical data can typically be retrospectively collected.

Scenario 4: data are available on a control area postlaunch as well as the intervention area data

Scenarios 1-3 describe when data are only available for the area covered by the intervention. However, data are often available for comparator areas as the informative outcome is often routinely collected and available across multiple areas, through systems such as Hospital Episode Statistics (HES) or collection can be prospectively arranged. Such comparator areas can be local, regional, national or a synthetic comparator created by combining a number of areas. To be an informative comparator, the area must represent a good match to the intervention area in all relevant characteristics and not be impacted by the launch of the new service being evaluated. ${ }^{5}$ The goodness of the match can be determined qualitatively or quantitatively by comparing the known features of the two areas.

The most common use of such control data is to directly compare the postintervention outcomes in the two areas, using the same approach as scenario 3, but with the contemporary control data are used instead of the historical intervention area data. As before, there are two categories:
A. Only aggregate data are available postintervention launch for the two areas. As in previous scenarios, an example of this would be analyses based on audit data alone but now across multiple areas.

B. Disaggregated data are available postintervention, allowing a disaggregated matched comparison can be made which again, results in the same total estimated impact as part A but gives us an understanding of the respective trends. This situation would occur where intervention is only launched in one part of a larger geographic area or patient group where the decisionmakers have access to the data of the full set prospectively, for example, one GP practice in a CCG area.

Under this scenario, comparator area data are used either instead of, or due to a lack of, historical evidence as used in scenario 3. Using simple analytical techniques, there is no way to incorporate both, which we will explore in scenario 6. There is no definitive rule for whether historical or contemporary comparator evidence is more appropriate, it is situation dependent. For example, if the intervention of interest was not the only change at the point of launch of the intervention, the control area data would likely be most appropriate if the second new service was launched in both areas, but not if it were only in the control area. A number of other factors must be considered, for example, what if comparator data are available but is not a good match, how does one define a suitable match, and what if there are multiple comparators potentially telling different stories?

\section{Scenario 5: all prelaunch and postlaunch data are available for the intervention area}

In this scenario and scenario 6 , we explore the addition of more advanced analytical approaches to the analysis of the data, specifically the use of interrupted time series (ITS) or 'segmented regression' analysis. This approach has been well presented in the literature, ${ }^{5-7}$ but in brief, the method considers the trend in an outcome of interest over time, segmenting it into the period before the intervention was launched, and after it. The example of using prelaunch and postlaunch data for the intervention area is shown in the explanatory figure 1 , where the prelaunch data are used to infer a postlaunch counterfactual case, with the nature of the change in the outcome define a priori. Using

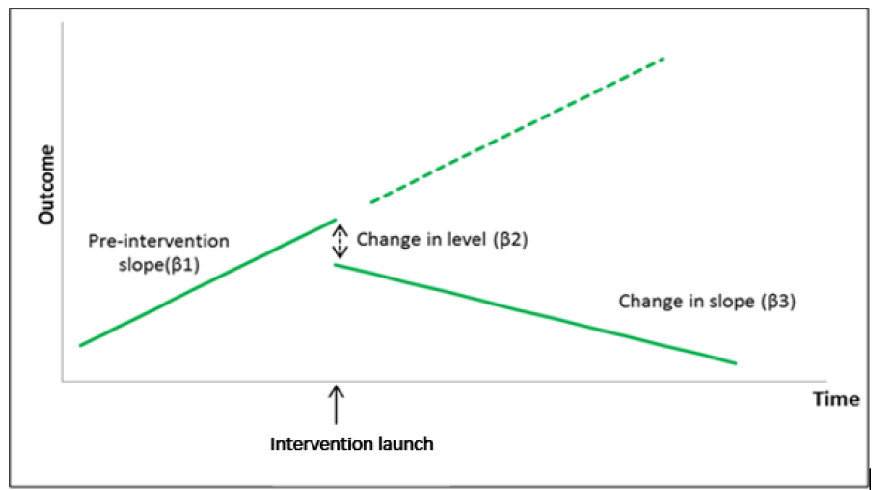

Figure 1 ITS analytical method. ITS, interrupted time series. 
the framework described by Bernal $e t a l^{7}$ it is possible to define the regression model using the equation detailed below, where $Y$ is the aggregated outcome, $\beta$ represents the relevant coefficients, $T$ the time since the start of the study, $t$ the specific time point, $X$ is a dummy variable indicating when the new intervention is active and $\varepsilon$ the error term.

$$
Y_{t}=\beta_{0}+\beta_{1} T_{t}+\beta_{2} X_{t}+\beta_{3} X_{t} T_{t}+\varepsilon_{t}
$$

The application of such a regression model allows for the formal estimation of whether any change in the outcome of interest is statistically significant under a frequentist framework and for any change to be quantified by estimating the area between the two regression lines, shown in figure 1 , over the analysis period.

The use of such a method requires time series data both before and after the launch in the intervention area, as in scenario 3B.

\section{Scenario 6: data are available on both control and intervention areas prelaunch and postlaunch}

We demonstrated in scenario 4 that the addition of control area data typically implied the exclusion of historical intervention area data in informing the counterfactual. Using ITS methodology, it is possible to formally incorporate comparator data, potentially from multiple areas or a synthetic area, alongside the full set of intervention area data. The method uses the preintervention data to formally test whether the comparator areas can be considered a good match. If so, the postlaunch comparator data are then used to infer the postlaunch counterfactual of the intervention area. Therefore, this approach assumes that the control area is indicative of what would have happened to the outcome in the intervention area had the launch not occurred, much as we assumed in scenario 4 but with a formal assessment of the trend and reliability of the comparator. The equation detailed in scenario 5 can be extended by incorporating a $\mathrm{Z}$ term as a dummy for assignment to the treatment or control population, as detailed by Linden ${ }^{5}$ :

$$
\begin{aligned}
Y_{t}= & \beta_{0}+\beta_{1} T_{t}+\beta_{2} X_{t}+\beta_{3} X_{t} T_{t}+\beta_{4} Z+ \\
& \beta_{5} Z T_{t}+\beta_{6} Z X_{t}+\beta_{7} Z X_{t} T_{t}+\varepsilon_{t}
\end{aligned}
$$

\section{Comparing the scenarios}

Each of the scenarios outlined above is characterised by a set of core assumptions, made implicitly or explicitly if used to evaluate the impact of a new intervention on some outcome of interest. Similarly, the variability in the ease of implementation, and data and analytical requirements of each scenario implies a range of pros and cons associated with each. These are presented in table 1, which highlights that the more analytically simple and data light the scenario the stronger the core assumption required about the nature of the interaction with the outcome and time trends in the data.

\section{Case study}

To explore the practical implications of the different scenarios and demonstrate the potential for varied conclusions, we have created a case study to which each is applied. To inform the case study, a time series dataset of an outcome unit of interest (eg, bed days, hospital admissions or indicators of quality and care outcome) has been simulated. The data values and number of time points have been selected to best inform the characteristics of each of the scenarios described in table 1 while representing the uncertain nature of real-world data relevant to this setting.

These data relate to two distinct groups (intervention and control) and a maximum of 30 observations are available over some defined time period at regular intervals (eg, every week, month or year). The data are structured such that in both areas, the outcome was increasing for the first 15 observations at a rate of $4 / 3$ per time period from a mean value of 20 units at time 1, after which point, the intervention is implemented in the intervention area but not the control. From time point 15 onwards in the intervention area, the outcome decreases at the same rate of 4/3 units per period, whereas in the control area, the outcome levels off, assumed to be due to factors unrelated to the intervention. All time points are subject to some level of variation to mimic what is observed in real-world data, simulated using a normal distribution (mean $=45$ and $\mathrm{SD}=5)$. We assume that after launch $(\mathrm{t}=15)$, the new service becomes fully operational, with no run-in period. The last time point in the intervention area $(t=30)$ was set as an extreme outlier (estimated as occurring with a probability of 0.99999 on the simulated distribution) to explore its impact on the results, for example, if an exogenous factor affected the intervention such as failure of a key piece of machinery. Figure 2 shows the fabricated data in full, with each data point representing the time period before, such that data point 1 being the total outcome over time 0 to 1 .

Using the informative structure of the simulated case study, it is possible to estimate two possible underlying effect values. If the control area is the best indicator of the counterfactual, then the intervention resulted in a reduction of 151 units over the period, if the historical intervention area is best, then a reduction of 324 units. Although these values can help us to understand the results of the different scenarios, they must be interpreted with caution; as while they inform the underlying trend used to simulate the data, the case study time points were sampled independently.

In the next part, we explore what the data availability would look like under each of the scenarios outlined in the previous section, estimating what the impact and conclusions would be regarding the effectiveness of the intervention. As outlined earlier, in many of the cases, only a limited set of the data are available, indeed it is only scenarios 4 and 6 where the full dataset is available to the decision-maker. Figures 3 and 4 provide an overview of the data availability across all of the scenarios.

Table 2 gives an overview of the results of the different possible scenarios and possible interpretations.

Figures 3 and 4and table 2 demonstrate the large potential for variation in the estimated impact of the 
Table 1 Summary of the different analytical methods

\begin{tabular}{llll} 
Method & Core assumptions & Pros & Cons \\
\hline $\begin{array}{l}\text { Scenario 1, only data } \\
\text { after launch in the } \\
\text { intervention area }\end{array}$ & $\begin{array}{l}\text { Only the change in the data } \\
\text { after the launch is relevant to } \\
\text { the evaluation }\end{array}$ & $\begin{array}{l}\text { Requires little data or technical } \\
\text { knowledge }\end{array}$ & $\begin{array}{l}\text { Unable to comment on the change } \\
\text { in the outcome of interest because } \\
\text { of the intervention, only its trend } \\
\text { after launch }\end{array}$ \\
$\begin{array}{l}\text { Scenario 2A, first } \\
\text { and last time point of } \\
\text { intervention period }\end{array}$ & $\begin{array}{l}\text { The two data points are fully } \\
\text { indicative of the change }\end{array}$ & $\begin{array}{l}\text { Requires little data or technical } \\
\text { knowledge }\end{array}$ & $\begin{array}{l}\text { Highly dependent on a small array } \\
\text { of data. } \\
\text { Risks loss of important details of } \\
\text { data, intervention effect or trends }\end{array}$ \\
$\begin{array}{l}\text { Scenario 2B, } \\
\text { disaggregated change } \\
\text { from starting period }\end{array}$ & $\begin{array}{l}\text { Last preintervention period fully } \\
\text { represents the counterfactual }\end{array}$ & $\begin{array}{l}\text { Only requires one } \\
\text { preintervention data point. } \\
\text { Analytically simple }\end{array}$ & $\begin{array}{l}\text { Highly dependent on a small array } \\
\text { of control data. } \\
\text { No consideration of trend in } \\
\text { counterfactual }\end{array}$
\end{tabular}

Scenario 3A, simple average of historical intervention area data

Scenario 3B, matched
preintervention and
postintervention

Simple averaging of before and after data incorporates all factors, there is no value in an assessment of the trends

There is a repeating periodic fluctuation, eg, seasonality, which impacts the outcome of interest and the trend over time is informative

The selected control area fully represents the counterfactual of the intervention area

The selected control area fully represents the counterfactual of the intervention area and the trend over time is informative

Scenario 5, ITS analysis Regression of preintervention of intervention area

Scenario 6, ITS
analysis of control and
intervention area

data fully represents postintervention counterfactual and the trend over time is informative

\section{Control area fully represents}

the counterfactual of the intervention area but the match can be tested by exploring the preintervention data. The trend over time is informative
Only requires a small amount Fails to explore trends in data of pre and post data.

Analytically simple

\section{Simple means of adjusting for Result varies given matching periodic fluctuations \\ approach.}

Blunt means of adjusting for periodic fluctuations that can result in incorrect estimates

Allows for use of control area

Fails to explore trends in data. Makes no use of historical data.

Only requires postlaunch data Difficult to determine if the contro area represents a reasonable comparator

Allows for use of control area Makes no use of historical data. data. Difficult to determine if the control Explores trends in data without area represents a reasonable having to define a cycle length. comparator Only requires postlaunch data

Allows for use of historical control data.

Explores the trends

Reliant on historical intervention area data being predictive of counterfactual

\section{Allows for use of control area and exploration as to the closeness of the control and intervention areas}

Assumption that the control area continues to represent a good match after the intervention period is a very incorrect conclusion. However, the appropriateness of the scenarios and accuracy of their conclusions compared with any 'true' effects are clearly much harder to determine in the real world.

\section{DISCUSSION}

In this paper, we have explored a range of possible scenarios and analytical approaches available to a decisionmaker when evaluating the impact of a new intervention on an outcome of interest, highlighting the implicit data and knowledge of the underlying trends in the simulated data, it is clear that several of these scenarios result drawn given the different scenarios. Estimations of the change in the outcome vary from predicting the interveneriods, as well as the overall impact of the intervention.

In the case study presented here, with full access to the 


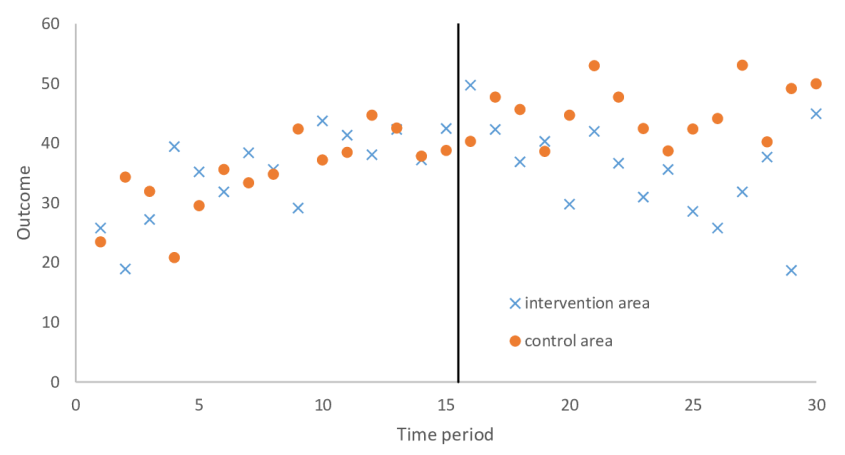

Figure 2 Fabricated time series data.

assumptions made in each. Through our simulated case study, we have demonstrated how these scenarios can yield very different estimates of effectiveness.

A comparison of the methods explored here suggests that it is intuitively appealing to conclude that the approach outlined in scenario 6, using the ITS methodology including the control area comparison, is the most

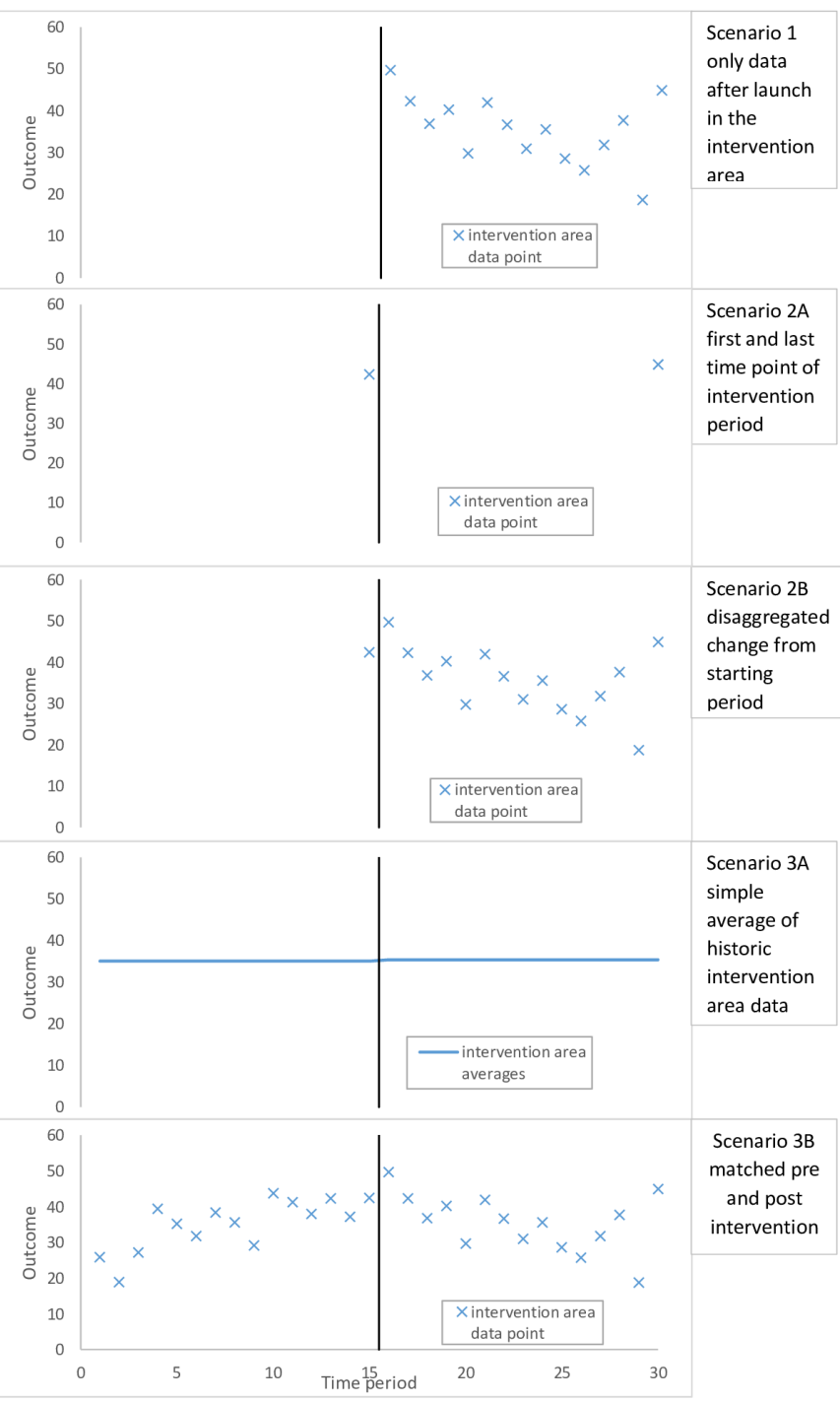

Figure 3 Data availability across the different scenarios of the case study, scenarios 1-3.

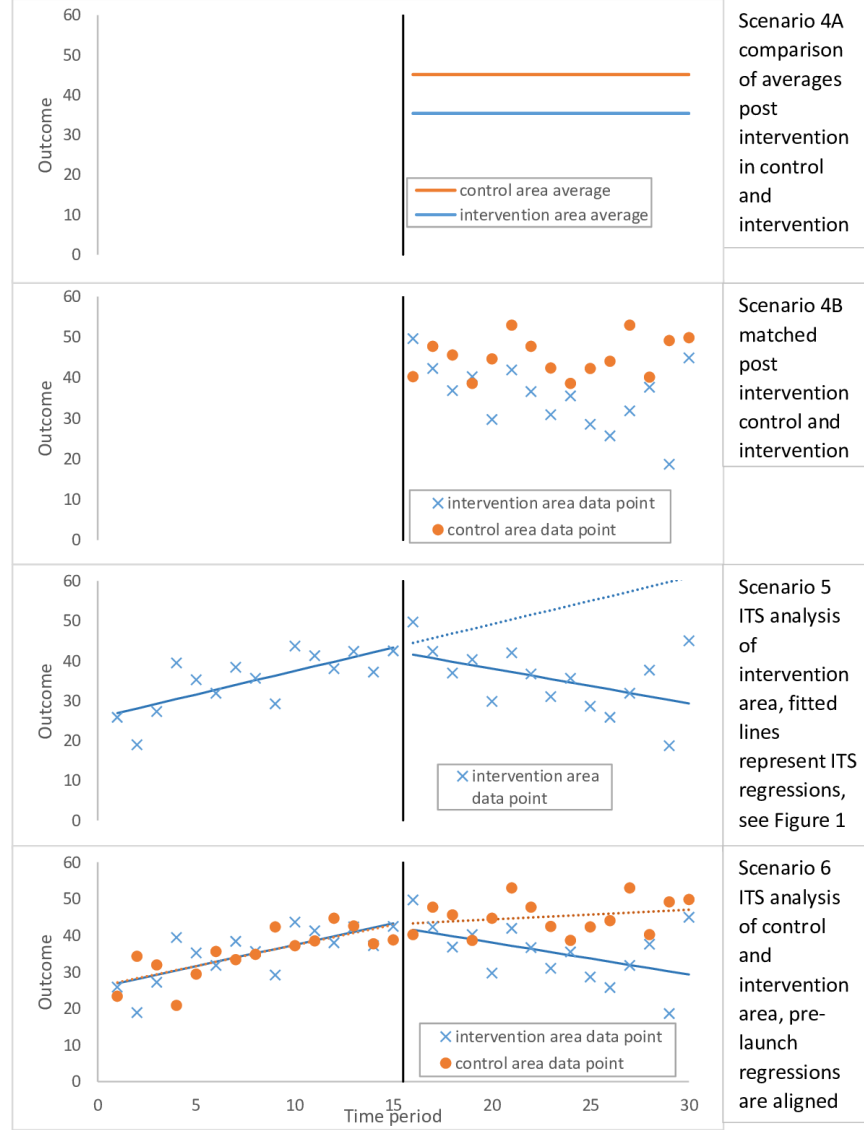

Figure 4 Data availability across the different scenarios of the case study, scenarios 4-6. ITS, interruptedtime series.

accurate as it incorporates the most complete set of data while taking the most complete approach to statistical analysis. However, the most appropriate methodology may be driven by other factors, primarily the availability of informative data and the validity of the core assumptions detailed in table 1.

Furthermore, the use of ITS analysis (scenarios 5 and 6 ) is not without assumptions, primarily relating to the suitability of the historical and control area data to inform the counterfactual and the functional form of the trends modelled. It also requires a significant level of data and analytical ability to implement. However, the inability to observe exactly what would happen in the intervention area without the new service necessitates such assumptions in order to estimate the impact of its launch. Fears about the robustness of such assumptions are likely to be best addressed by the identification of additional relevant evidence to either adjust the existing data or inform a new comparator. For example, methods are available to overcome concerns over additional service changes in the time period covered by the data, ${ }^{5}$ to incorporate multiple control areas ${ }^{5}$ and to conduct a more rigorous selection of control area through matching. ${ }^{8}$

As with all such analyses, the ITS methodology can be extended to consider the significance of the findings beyond pure chance. This can be achieved through a frequentist framework, considering the statistical significance of the 
Table 2 Summary of the different scenarios results

$\begin{array}{lll}\text { Scenario } & \text { Possible interpretation of the result } & \text { Estimated change* }^{*} \\ \begin{array}{ll}\text { Scenario 1, only data after launch in } \\ \text { the intervention area }\end{array} & \begin{array}{l}\text { The outcome of interest appears to have decreased } \\ \text { over the postlaunch time period }\end{array} & \begin{array}{l}\text { Not possible to estimate a } \\ \text { change in the outcome }\end{array}\end{array}$

Scenario 2A, first and last time point There appears to have been an increase in the outcome 37.6

of the intervention period

from the prelaunch to postlaunch period. Extrapolating

the observed values over the entire 15 months of

intervention suggests that the new intervention had

increased the outcome by 37.6 units $((44.9-42.4) \times 15)$

Scenario $2 \mathrm{~B}$, disaggregated change
from starting period

The outcome of interest appears to have decreased

$-120.1$

over time from the prelaunch time period, with an

estimated change of -120.1 units over the period

((34.4-42.4)x15)

Scenario 3A, simple average of historical intervention area data

Scenario $3 \mathrm{~B}$, matched
preintervention and postintervention

There appears to have been little change from the prelaunch to postlaunch periods in the outcome, with the average value going from 35.1 to 35.4 ((35.4-35.1)x15)

\section{There appears to have been little change from the} prelaunch to postlaunch periods in the outcome, with the average value going from 35.1 to 35.4 . However, it appears from the data that there was an increasing trend in the outcome before the intervention and a decreasing trend afterwards ((35.4-35.1)x15)

Scenario 4A, comparison of Compared with the control area the intervention area $-146.0$ averages postintervention in control had a lower average level of the outcome after the and intervention areas

Scenario $4 \mathrm{~B}$, matched
postintervention control and
intervention area
launch of the intervention

Compared with the control area, the intervention area
had a lower average level of the outcome after the
launch of the intervention. The control area appeared
to have a flat trend in the outcome over the postlaunch
period compared with a decreasing trend in the
intervention area
$((35.4-45.1) \times 15)$

Scenario 5, ITS analysis of intervention area

Compared with the prelaunch intervention area the postlaunch saw a decrease in the trend over time in the outcome, from positive to negative, which was statistically significant.

See the online supplementary appendix for regression

\begin{tabular}{|c|c|}
\hline $\begin{array}{l}\text { Scenario } 6, \text { ITS analysis of control } \\
\text { and intervention area }\end{array}$ & $\begin{array}{l}\text { Both control and intervention areas saw a shallowing of }-146.0 \\
\text { the trend over time. The intervention area saw a greater } \\
\text { decrease in the trend, being negative compared with } \\
\text { the relatively flat trend in the control. This difference } \\
\text { was statistically significant. The control area was found } \\
\text { to be a match to the intervention area in the prelaunch } \\
\text { period (the regressions lines are aligned). See the } \\
\text { Supplementary Appendix for regression }\end{array}$ \\
\hline
\end{tabular}

*Negative values indicate that the new service reduced the outcome. ITS, interrupted time series.

regression estimates, as discussed in Linden, ${ }^{5}$ through a Bayesian framework. ${ }^{9}$ Such considerations should play an important role in the decision-making process, as a single estimate of the impact on an intervention can be misleading. Specifically, it fails to take account of the uncertainty, of the informative data or the consequences of making an incorrect funding decision. However, it is important to reflect that even if there is substantial uncertainty, it is the expected impact of the intervention that should be most informative to the commissioning decision, rather than the significance of the impact. ${ }^{10}$

An intrinsic element to any analyses explored in this paper is an understanding of the data under interrogation: the application of robust methods is only helpful if the data being used are consistent and relevant to the question being addressed. Prior to any analysis, it is important to 
understand the data, answering questions such as: how was it generated; is an estimate of the rate of an event more relevant than its frequency; is it consistent over the time period of interest; what is the route of causality between the intervention of interest and the data; and when plotted do there appear to be any unexplainable outliers?

Analyses such as those presented here are most robust when combined with qualitative methodologies through a mixed-method approach, with the qualitative findings ideally facilitating a more detailed understanding of the trends seen in the data and informing the suitability of the different counterfactual scenarios. Such a mixed-methods approach may extend the quantitative incorporate health economic considerations, such that the generalisable costeffectiveness of the intervention is considered.

Furthermore, the use of robust methodologies, such as ITS analysis, does not replace the need for the robust selection of outcomes and data collection, as any analysis can only be as robust as the data that informs it. Failure to prospectively design the launch of intervention and associated evaluation to ensure, the required level of data collection and sufficient consideration of a contemporaneous control will likely lead to an erroneous result whatever evaluative method is used.

\section{Patient and public involvement}

As the informative dataset was simulated, there was no patient nor public involvement in this study, nor was consent required for access to patient data.

Contributors SH: devised the idea for the paper, generated the informative data, conducted the analysis and led the drafting of the paper. LB and GR: provided recommendations on the generation of the data and the analysis, in addition to contributing to the drafting of the paper.

Funding This article presents independent research by the National Institute for Health Research Collaboration for Leadership in Applied Health Research and Care Yorkshire and Humber (NIHR CLAHRC YH) and the NIHR Applied Research Collaboration Yorkshire and Humber (ARC YH).

Competing interests None declared.
Patient consent for publication Not required.

Provenance and peer review Not commissioned; externally peer reviewed.

Data availability statement All data relevant to the study are included in the article or uploaded as supplementary information.

Open access This is an open access article distributed in accordance with the Creative Commons Attribution Non Commercial (CC BY-NC 4.0) license, which permits others to distribute, remix, adapt, build upon this work non-commercially, and license their derivative works on different terms, provided the original work is properly cited, appropriate credit is given, any changes made indicated, and the use is non-commercial. See: http://creativecommons.org/licenses/by-nc/4.0/.

ORCID iD

Sebastian Hinde http://orcid.org/0000-0002-7117-4142

\section{REFERENCES}

1 Health and social care act 2012. Available: http://www.legislation. gov.uk/ukpga/2012/7/contents/enacted [Accessed 31 May 2018].

2 NHS Research and Development Forum. Bath Research \& Development, Research, evaluation and evidence: a guide for commissioners. Available: http://www.rdforum.nhs.uk/content/ wp-content/uploads/2016/04/GUIDE-Evaluation-and-Research-forCCGs-Final-version-April-2016.pdf [Accessed 31 May 2018].

3 NHS England. The better care fund, how to. understand and measure impact, 2015. Available: https://www.england.nhs.uk/wp-content/ uploads/2015/06/bcf-user-guide-04.pdf.pdf

4 NHS England. NHS England, Evaluation strategy for new care model vanguards, 2016. Available: https://www.england.nhs.uk/wp-content/ uploads/2015/07/ncm-evaluation-strategy-may-2016.pdf [Accessed 31 May 2018].

5 Linden A. Conducting interrupted time-series analysis for single- and multiple-group comparisons. Stata J 2015;15:480-500.

6 Cruz M, Bender M, Ombao H. A robust interrupted time series model for analyzing complex health care intervention data. Stat Med 2017;36:4660-76.

7 Bernal JL, Cummins S, Gasparrini A. Interrupted time series regression for the evaluation of public health interventions: a tutorial. Int J Epidemiol 2017;46:348-55.

8 Linden A. Improving causal inference with a doubly robust estimator that combines propensity score stratification and weighting. J Eval Clin Pract 2017;23:697-702.

9 Spiers G, Allgar V, Richardson G, et al. Transforming community health services for children and young people who are ill: a quasiexperimental evaluation. Health Serv Deliv Res 2016;4:1-222.

10 Claxton K. The irrelevance of inference: a decision-making approach to the stochastic evaluation of health care technologies. J Health Econ 1999;18:341-64. 